\title{
Assessing the Relationship between the Economic Feasibility Study and the Guarantees Provided by Small and Medium Enterprises with the Financing Decisions in Kuwait
}

Yausef Alremaidhi ${ }^{1 *}$, Abdulmajid Obaid Hasan Saleh ${ }^{2}$, Anwar Hasan Abdullah Othman ${ }^{3}$

${ }^{1,2,3}$ International Islamic University Malaysia, Institute of Islamic Banking and Finance,

Kualalumpur, Malaysia

Email: Yoseif811@gmail.com, alamri@iium.edu.my, anwarhasan@iium.edu.my

Received March, 2020; Accepted May, 2020

\begin{abstract}
This paper sheds light on the role of Kuwaiti Islamic banks. It addresses banks' major requirements to access financing sources in the context of small and medium enterprises (SMEs). Narrowly, it weighs the impact of two bank requirements: the feasibility studies and the collateral, and pinpoints their effects on easing the process of SMEs borrowing. The study applies the quantitative approach. It aims to assess SMEs internal factors that might affect the Islamic banks' financing process and decision. The sample consists of 200 employees from the credit department of Kuwaiti Islamic banks. Results indicate a significant and positive association between the feasibility study indicator and the financing decisions on one hand and the collateral indicator and the financing decisions on the other hand. The study recommends Kuwaiti Islamic banks allocate more importance on the feasibility studies as a medium to access adequate financing resources.
\end{abstract}

Keywords: Small and Medium Enterprises; Feasibility Study; Guarantees; Financing Decisions; Islamic Banks; Kuwait

Type: Research paper

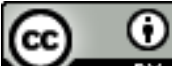

EY This work is licensed under a Creative Commons Attribution 4.0 International License.

DOI: 10.51325/ijbeg.v3i2.27

تقييم ارتباط دراســة الجدوى الاقتصـادية والضـمانات المقدمة بقرارات التمويل للمثـاربع الصـغيرة والمتوسـطة في دولة الكويت

الملخص:

يهدف هذا البحث إلى قياس ارتباط كلا من دراســة الجدوى الاقتصــادية والضــمانات المقدمة للتمويل المشــاريع الصـغيرة والمتوسـة وقرارات التمويل من المصـارف الاسـلامية في دولة الكويت. اتبعت هذه الدراسـة نوع الدراسـات الميدانية المتضــافرة بالأسـلوب الوصـفي التحليلي، مسـتهذفا تقييما صـحيحا للمتطلبات الداخلية لإقامة المشـروعات الصـــغيرة والمتوســـطة، والوقوف على الآثار المترتبة من هذه المتطلبات على القرارات التي تتخذها المصـــــارف الإسـلامية لتقديم تمويلات لهذه المشـروعات. شملت عينة الدراسـة 200 موظف من مجموع العاملين في المصـارف الإسـلامية ضـمن دائرة الإئتمان. أظهرت النتائج وجود تأثير ذو دلالة إحصـائية عند مستوى دلالة (0.05) لدراسـة الجدوى الإقتصـادية للمشـروعات الصـغيرة والمتوســة على قرارات تمويل هذه المشـاريع من المصـارف الإسـلامية الكويتية. كما اظهرت وجود تأثير ذو دلالة إحصائية عند مستوى دلالة (0.05) للضمانات المقدمة من المشروعات الصــيرة والمتوســة على قرارات تمويل هذه المشـاريع من المصــارف الإسـلامية الكويتية. توصــي الدراسـة قيام المصسارف الإسـلامية الكويتية بالمسـاهمة في إعتماد دراسات الجدوى الاقتصـادية للمشروع الصـغير والمتوسط وذلك للوقوف على إمكانية نجاحه في المستقبل ولتقليل الضمانات المطلوبة لتمويله من قبل هذه المصارف. 
الكلمات المفتاحية: المشـاريع الصـغرى، دراسـة الجدوى الاقتصـادية، الضـمانات المقدمة، البنوك الاسـلامية، قرارات التمويل، الكويت

المقدمة

تلعب المشاريع الصغرى دوراً ايجابياً في دعم حركة التتمية الاقتصادية وزيادة النمو الاقتصادي ورفع مستوى الدخل

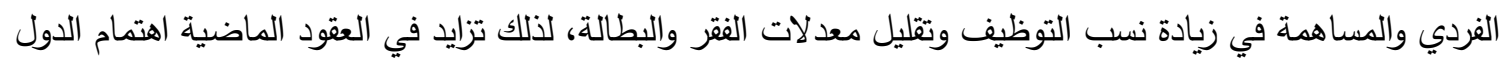

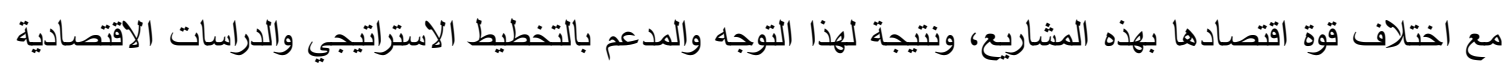

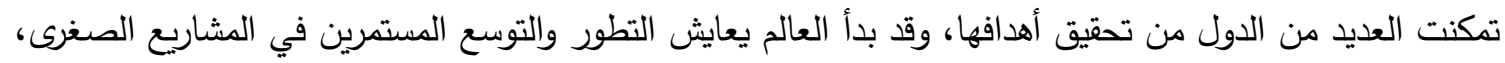

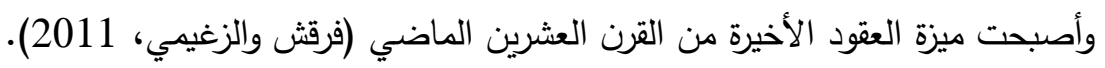
كما أن التقدم الاقتصادي الذي تسعى إليه كافة اقتصاديات بلدان العالم أخذ يعتمد إلى حد كبير إلى نمو واتساع

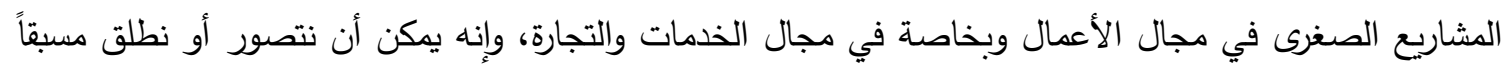

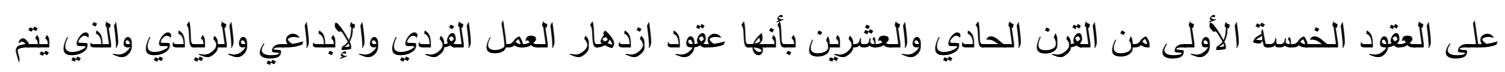

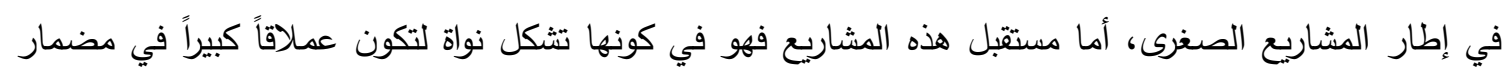

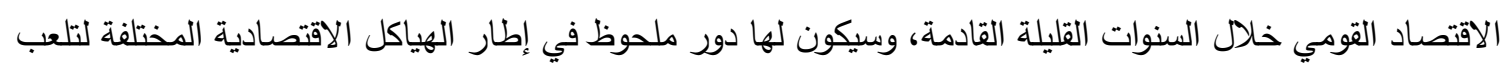
دورها وتتحمل مسؤولياتها (جواد والمنصور ، 2017).

لذلك باتت المشاريع الصغرى تتمتع بأهمية كبيرة نظرا للخصوصيات التي يكتسبها هذا النوع من المشاريع من صغر

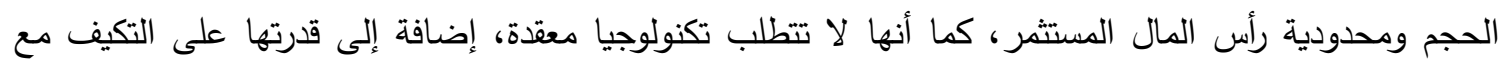

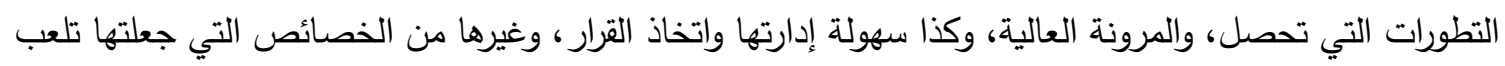
دوراً كبيراً من تثغيل الأيدي العاملة والتقليل من نسبة البطالة.

كذلك فان المشاريع الصغرى في مختلف القطاعات التي تتتمي إليها تحتاج إلى ظروف مواتية للعمل وإلى أنثطة وآليات تدعم عملياتها على الأقل في السنوات المبكرة من دورة حياتها، إذ تعجز هذه الأعمال والمشاريع على مواجهة

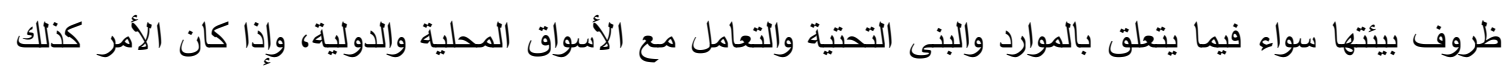
للمشروعات القائمة، فالحال سوف يغدو أكثر صعوبة أمام الجديدة منها.

لذلك فان الإبداع والابتكار يعتبران المحرك الرئيسي للنمو والتتمية في المجتمع، وبالنظر إلى المستويات المرتفعة من

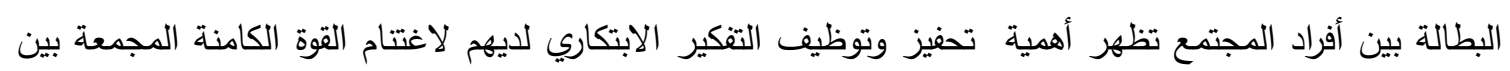

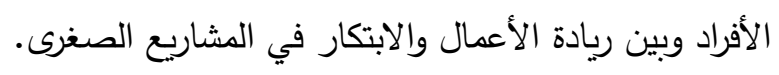

مشكلة البحث

تمثل المشاريع الصغرى والمتوسطة العصب الرئيسي والهام لاقتصاديات الدول، خصوصا تلك التي تثح فيها الموارد الطبيعية، ذلك نظرا لقدرة هذه المشاريع على اضافة التتوع الاقتصادي للمنتجات والخدمات المقدمة. يعتمد اقتصاد

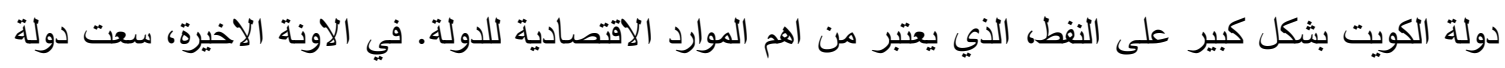

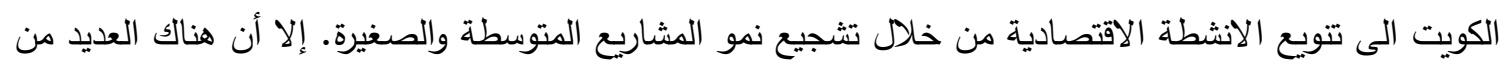

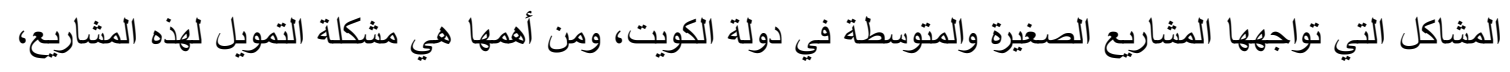


إذ أن استمرار هذه المشاريع وبقاءها يعتمد بشكل أساسي على مدى توفر مصادر تمويل مناسبة من أجل ضمان بقاء

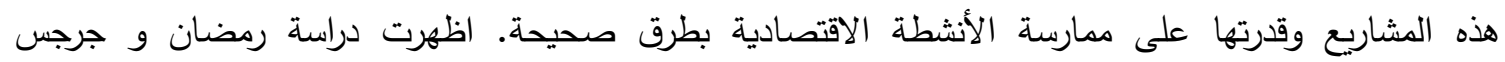
(Ramadhan \& Girgis, 2018) بدول الخليج، يعزى السبب في ذلك الى عدم الوفاء بمتطلبات الحصول على التمويل المناسب لاقامة هذه المشاريع. حيث يعد تقديم دراسات الجدوى غير المكتملة من احد اهم المتطلبات التي تثكل عائقا هاما للحصول على التمويل.

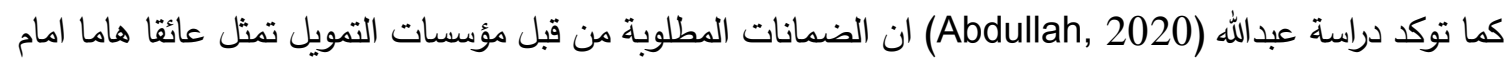
تطور قطاع المشاريع الصغيرة والمتوسطة في الكويت. اظهرت دراسة عبدالله ان مايقارب 18\% من المشاريع التي مؤسيات يتم رفضها يعود الى ضعف الضمانات المقدمة للحصول على التمويل. بناء على مشكلة الدراسة، تهدف الدراسة

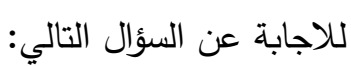

- ما مدى ارتباط كلا من دراسة الجدوى الاقتصادية والضمانات المقدمة بقرارات التمويل للمشاريع الصغيرة

$$
\text { والمتوسطة من قبل المصارف الاسلامية الكويتية }
$$

\section{مفهوم المشاريع الصغرى}

لقد وجدت المشاريع الصغرى بفعل المبادرات الفردية أو الجماعية بهدف تحقيق العوائد والأرباح، وذلك من خلال تقديم

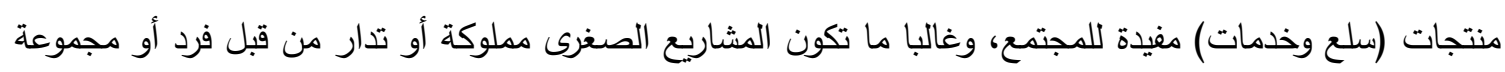

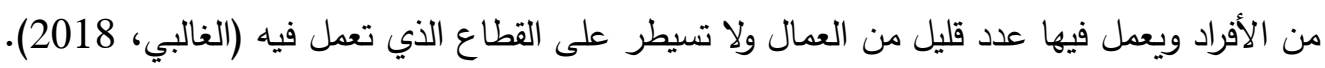

وفي إطار هذه المشاريع الصغرى هناك العديد من النشاطات في مختلف القطاعات، كما أن صيخ كثيرة من الأعمال

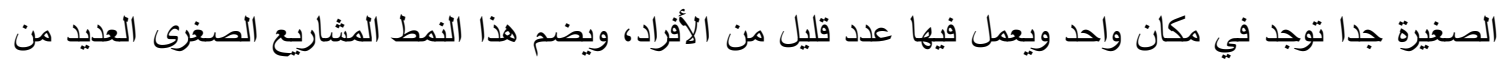
الأعمال الحرفية في مختلف القطاعات والتي تعتمد على الموارد المحلية وغالبا ما تسوق مخرجات ومنتجات هذئ هذاته

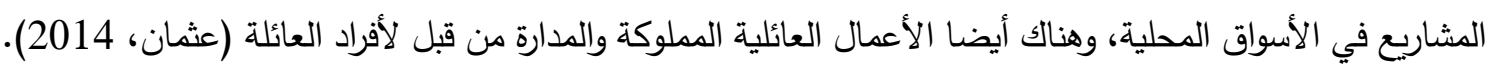

تم تعريف المشروعات الصغرى بأنها " منظمات أو كيانات قانونية رأسمالها صغير يقوم بتأسيسها فرد واحد أو مجموعة

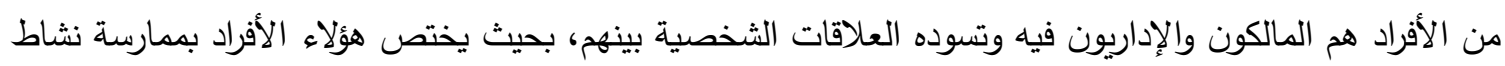

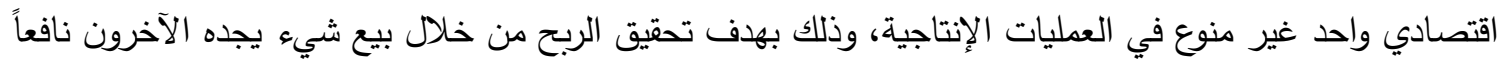
فيدفعون ثمناً له (بودرامة ومهملي، 2010).

ويعرف الباحث المشروعات الصغرى على أنها المشروعات التي عادة ما تكون إدارتها مستقلة، أي أن مالك المشروع هو المدير في الوقت ذاته، وان هناك شخص واحد أو مجموعة شركاء يقدمون رأس المال وهم المالكون عادة لمثل عادي

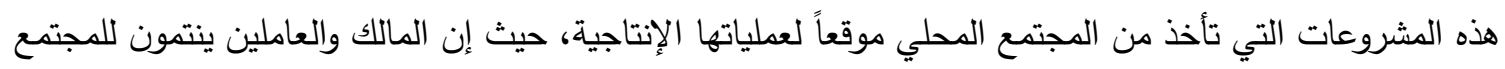

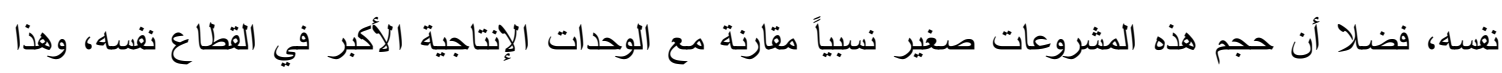
المعيار يحدد وفق حجم المبيعات أو عدد العمال أو رأس المال.

\section{أهمية المشاريع الصغرى}

تُعد المشاريع الصغرى دعامة أساسية من دعامات الاقتصاد الوطني كونها تسهم في زيادة معدلات النمو الاقتصادي، وتعمل على خلق القيمة المضافة في إنتاج السلع والخدمات، وفي استغلالها للموارد الإنتاجية المتاحة، وكذلك إسهامها 
في الحد من البطالة والتضخم، وذلك من خلال توظيف الأموال والأفراد، ومواجهة تحديات الفقر ورفع المستوى

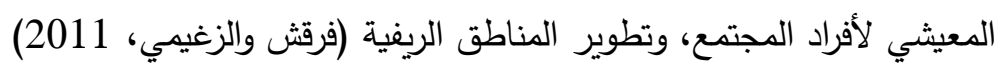

ويؤدي انتشار تثافة ريادة الأعمال وامتلاك المشاريع الصغرى إلى توفير فرص عمل مستدامة تسهم في معالجة

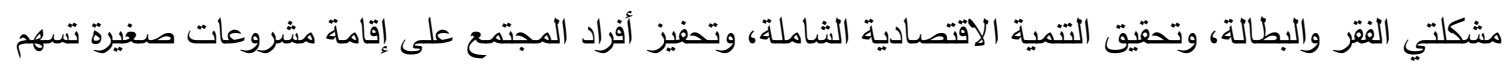
في بناء اقتصاد نوعي قادر على دخول الأسواق العربية والعالمية (أبو جليل، 2017).

كما تعمل المشاريع الصغرى على صقل المهارات الإدارية والفنية والسلوكية، من خلال الاستثمار في العنصر البشري،

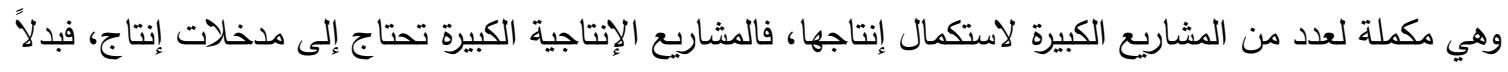

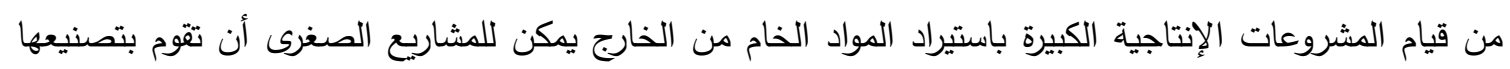

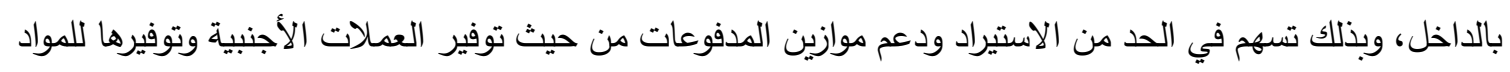

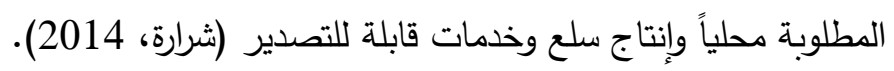

$$
\text { ويشير (أبو جليل وآخرون، 2017) إلى أن أهمية المشاريع الصغرى تتمثل فيما يلي: }
$$

1. تساهم المشاريع الصغرى في تتويع تثكيلة المنتجات سواء كانت سلع أو خدمات وتوسيعها كذلك في تتمية المدخرات الدحلية وإحلال الواردات، وذلك من خلال عرضها تلونا للسلع والخدمات في السوق المحلية بأسعار منافسة وجودة عالية.

2. تسهم المشاريع الصغرى في تتمية الصادرات من خلال التصدير المباشر للمنتجات التي تقوم بإنتاجها، وتوفير الصناعات المغذية والعالية الجودة والمنافسة بالسعر للصناعات الكبيرة التي تقوم بتصدير منتجاتها،

$$
\text { ومنافسة بعض المشروعات الإنتاجية الكبيرة. }
$$

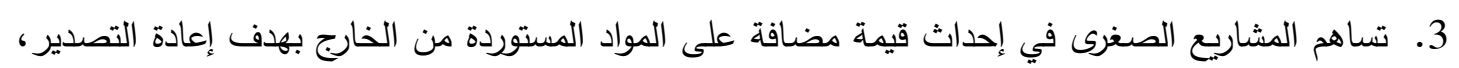

أي ممارسة نشاط إعادة التصدير الذي يؤدي إلى تكوين التراكم الرأسمالي واستخدام المواد الخام.

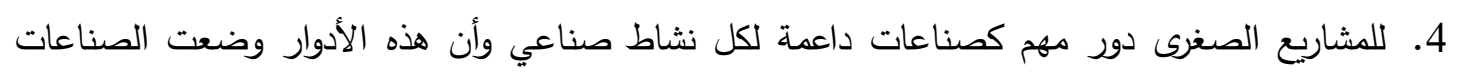

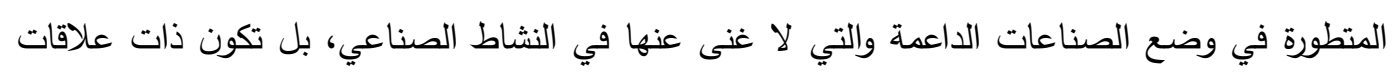
تعاقدية مع الصناعات الأخرى في الوقت نفساعه.

ويرى الباحث أن أهمية المشاريع الصغرى تكمن في أنها تسهم في تحريك عجلة النمو الاقتصادي وفي تحقيق أهداف

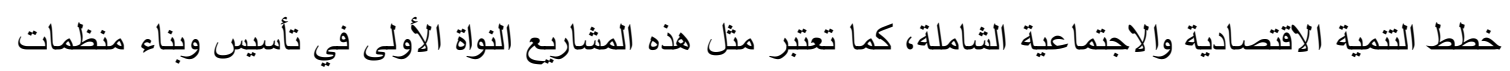
الأعمال الكبيرة وعلى مختلف مستوياتها التتظيمية، مما يجعل مثل هذه المشاريع قادرة على الدخول إلى الأسواق، وهذه الحقائق تجعلها من الحقول الهامة في اقتصاديات الدول الصناعية.

\section{المعايير الدولية والقوانين العربية في تصنيف المشاريع الصغرى}

إن المعايير التي يمكن الاستناد عليها لتحديد وتصنيف المشاريع الصغرى تختلف من دولة لأخرى وفق لإمكانياتها وقدراتها وظروفها الاقتصادية ومراحل النمو التي وصلت إليها، وبالتالي فان المشاريع التي تعتبر كبيرة الحجم في دولة التئل نامية يمكن اعتبارها صغيرة الحجم في دولة متقدمة، كما يظهر اختلاف معايير ومؤشرات القياس من دولة إلى أخرى، 
فعلى سبيل المثال: تعد المشاريع الإنتاجية صغيرة في ألمانيا إذا ما استخدمت لغاية 49 عاملاً، أما في الولايات

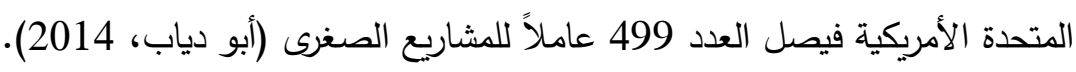
عرفت منظمة الأمم المتحدة للتتمية الصناعية (اليونيدو) المشاريع الصغرى بأنها تلك المشاريع التي يديرها مالك واحد

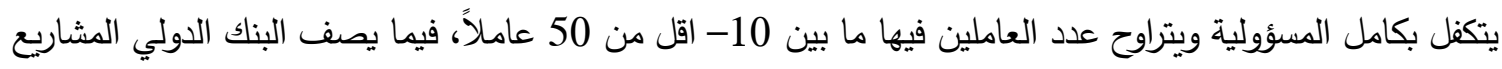
التي يعمل فيها اقل من 10 عمال بالمشاريع المتناهية الصغر ، والتي يعمل فيها بين 10-50 عاملا بالمشروعات الصغيرة، والتي يعمل فيها بين 50-100 عامل بالمشروعات المتوسطة (الثلبي، 2014).

ويشير (المحروق، 2011) إلى أن المشاريع الصغرى تتمتع بالعديد من الخصائص والمميزات، منها:

$$
\begin{aligned}
& 1 \\
& 2
\end{aligned}
$$

3 4

$$
\text { المحلية في تصريف منتجاتها. }
$$

5 6

7

8

9

10 سهولة الدخول والخروج من السوق.

11 الريادة والمبادرة.

12 توفير بيئة عمل ملائمة حيث يعمل صاحب المشروع الصغير والعاملون جنباً إلى جنب.

وحسب المفوضية الأوروبية فان المشاريع الصغرى تصنف إلى المشاريع المصغرة وتضم أقل من 10 أشخاص، والمشاريع الصغيرة وتضم من 10 إلى 49 شخص، والمشاريع المتوسطة وتضم من 50 إلى 249 شخص، ولصن والمشاريع الكبيرة وتضم أثر من 249 شخص (عثمان، 2014).

ويشير (الصرايرة، 2016) إلى إن المشاريع الصغرى في الدول العربية تقسم على أساس حجم النشاط إلى ما يلي: 1- الصناعات الصغيرة جداً (Adams \& Williams,2019; Alqallaf and Alareeni, 2018): التي تثغل اقل من 5 عمال وتستثمر أقل من 5000 دولاراً ( إضافة إلى استثمارات الأبنية والعقارات الثابتة ). 2- الصناعات الصغيرة (Darling et al.): التي تشغل 5-15 عامل وتستثمر أقل من إلى استثمارات الأبنية والعقارات الثابتة ).

3- الصناعات المتوسطة (Medium): التي تشغل 16-25 عامل وتستثر من 15000-25000 دولاراً (عدا الأبنية والعقارات). 
وفي اجتماع لتحديد تعريف موحد للمشاريع الصغرى عقد في العاصمة الأردنية (عمان) بتاريخ 2003/9/21، وحضره إثنا عشر مندوباً عن الجهات الحكومية وشبه الحكومية ومؤسسات تطوعية غير حكومية ذات علاقة بالمؤسسات الصغيرة، إذ أكد المشاركون في ذلك الاجتماع على عدم الأخذ برأس المال المسجل لغايات التعريف، لأن رأس المال

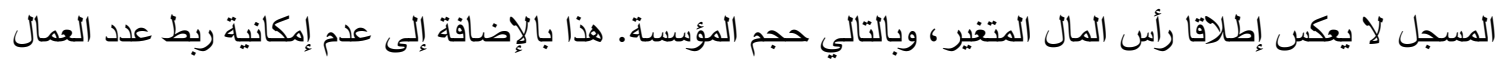
مع رأس المال المسجل، حيث توجد مؤسسات صناعية رأسمالها المسجل قليل بينما عدد عمالها كبير والعكس صحيح. وبناءً على ذلك تم تحديد واعتماد تعريف موحد للمشاريع الصغرى، وفقاً لعدد العمال واستثناء أي عامل آخر بما في ذلك رأس المال المسجل وعلى النحو التالي:

\begin{tabular}{|c|c|c|}
\hline عدد العاملين & التصنيف & $ت$ \\
\hline 1-4 عمال & مشروع متتاهي الصغر & 1 \\
\hline 5-19 عامل & مشروع صغير & 2 \\
\hline 20-99 عامل & مشروع متوسط & 3 \\
\hline أكثر من 99 عامل & مشروع كبير & 4 \\
\hline
\end{tabular}

أما الدليل الإرشادي للمؤسسات الصغيرة والذي نشرته منظمة الإسكوا عام 2003 فيصنف المؤسسات الصغيرة بناءً على حجم رأس المال المستثر وعدد العاملين وحجم المؤسسة وحجم الأرباح والمبيعات، ويميز ما بين المشاريع

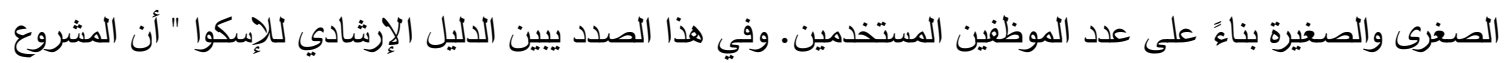
الميكروي لا يوظف أكثر من أربعة موظفين. أما المشروع الصغير فيوظف ما بين 4-10 موظفين، بينما يوظف ولف لهين المشروع المتوسط ما بين 10-25 موظفاًا.

ويختلف تعريف المشروع الصغير من دولة إلى أخرى ومن صناعة لأخرى فعلى سبيل المثال فإن صناعات الكمبيوتر تعد صغيرة إذا قل عدد الموظفين لايها عن 1000 موظف، وعن 1500 في الصناعات البترولية و 500 موظف لهن

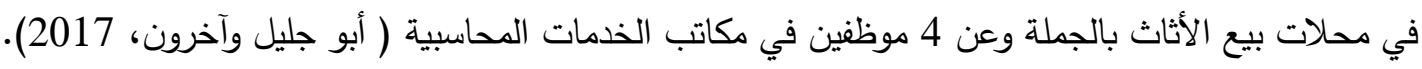
وقد كان تأسيس المشاريع الصغرى من أهم الأسباب المسؤولة عن النمو الاقتصادي في الدول المتقدمة خلال السنوات

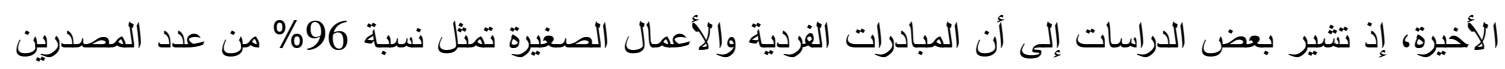
في الولايات المتحدة الأمريكية وقيمة 30\% من إجمالي الصادرات، كما ساهمت هذه المشروعات في خلق عدد كبير من فرص العمل في الاقتصاد الأمريكي تجاوزت 15 مليون فرصة عمل في مجال الخدمات عام الخدات 1992، كما أصبح

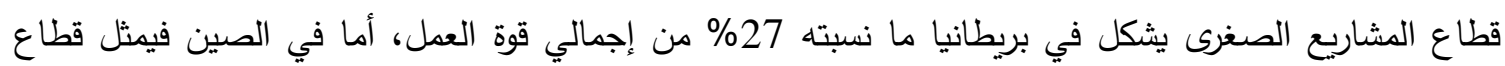
الأعمال الصغيرة أكثر من 50\% من الدخل القومي، وتعتمد بلاد كثيرة مثل سنغافورة وماليزيا واندونيسيا وغيرها، اعتماداً كبيراً في اقتصادها الوطني على مثل هذا النوع من المشروعات (القهيوي، 2015). 
كما تعتبر المشاريع الصغرى إحدى الركائز الأساسية للتتمية الاقتصادية ومن أهم استراتيجيات التتمية في الدول النامية

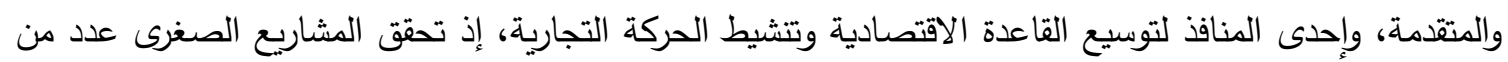
الوظائف الهامة المرتبطة بالكفاءة والتتافسية وخلق فرص العمل وابتكار المنتجات، إضافة إلى كونها تمثل رافداً أساسياً

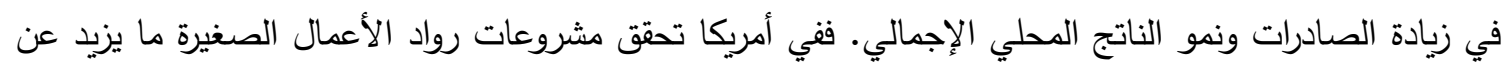

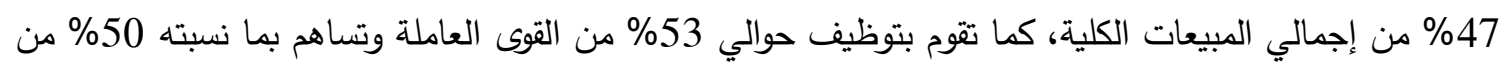

الناتج الإجمالي المحلي الأمريكي وتصل هذه المساهمة إلى 43\% في كوريا الجنوبية، و 56\% فئس \% في الصين و 70\% في هونك كونج (الصرايرة، 2016).

وتؤدي المشاريع الصغرى دوراً كبيراً في تحسين الوضع الاقتصادي للفرد، والتوظيف الذاتي لرواد الأعمال الصغيرة،

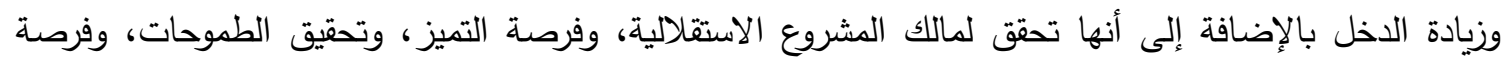

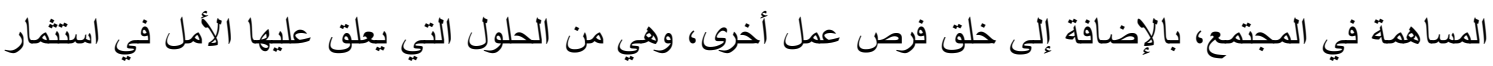
الفرص السكانية للكثير من الدول، كما إنها من الأساليب الحديثة التي أقبلت عليها العديد من الدئ الدول المتقدمة والنامية

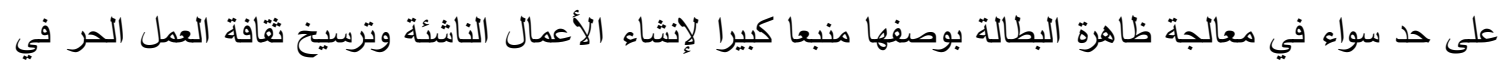

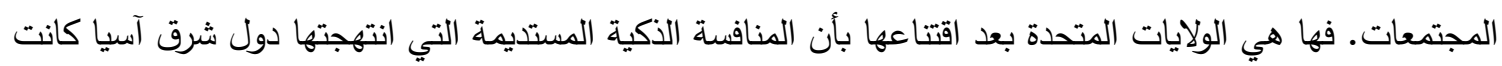

$$
\text { ترتكز إلى دعم رواد الأعمال والمشاريع الصغرى (المحروق ومقابلة، 2016). }
$$

وتأكيداً على الدور الهام الذي تساهم به المشاريع الصغرى، في الاستقرار والتتمية الاقتصادية والاجتماعية، فانه وحسب المفوضية الأوروبية تركز سياسة هذه المشاريع على خمسة مجالات ذات أولوية هي: (حمزة، 2015)

$$
\begin{aligned}
& \text { 1- تشجع روح المبادرة والمهارات الفردية. } \\
& \text { 2- تحسين وصول المشاريع الصغرى إلى الأسواق. } \\
& \text { 3- الحد من الإجراءات الإدارية. } \\
& \text { 4- تحسين إمكانات هذه المشاريع. } \\
& \text { 5- تعزيز الحوار والتشاور مع الأطراف المعنية. }
\end{aligned}
$$

ويرى الباحث أن منطقة الشرق الأوسط وشمال أفريقيا بما فيها الدول العربية، من المتأخرين نسبياً في تتفيذ سياسات وبرامج للحد من الحواجز أمام نمو المشاريع الصغرى على الرغم من إحراز تقدم مع إطلاق عدد من المبادرات لتعزيز

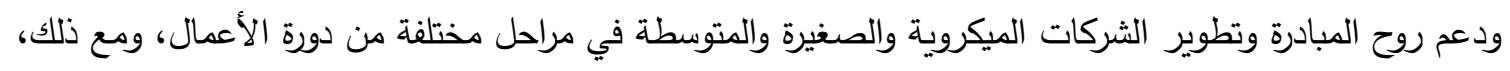

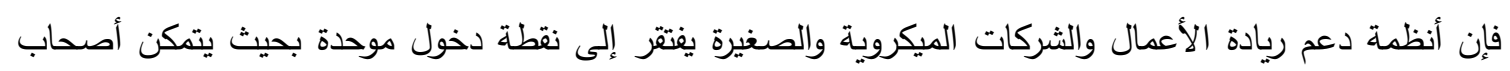

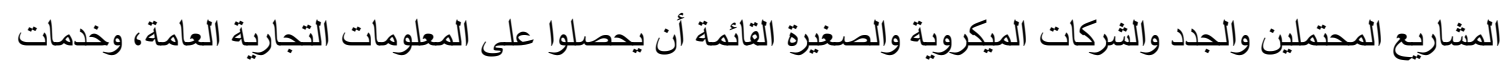
الدعم والإرشاد حول الإحالة والتعارف إلى مقدمي الخدمات المختلفة بناء على احتياجاتهم.

دور المشاريع الصغرى في التنمية الاقتصادية والاجتماعية تعتبر المشاريع الصغرى نواة للمشروعات المتوسطة والكبيرة، إذ أن هناك الكثير من هذه المشاريع استطاعت تحقيق

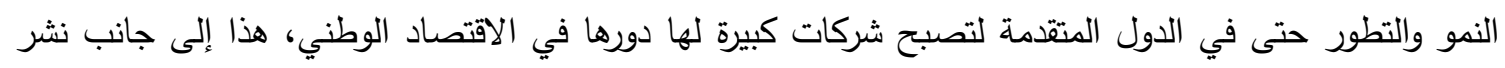
المعرفة التقنية اللازمة للمنثآت الأكبر ، إضافة إلى قدرتها على التخصص في مجال معين وبما يشابه الخط الإنتاجي 
الواحد أو جزء منه في منشأة أكبر، مما يعني إمكانية تحقيق تكامل الإنتاج في عدد من المنشآت الصغيرة وكأنه مجمع صناعي في منثأة أكبر .

وتؤدي المشاريع الصغرى دوراً كبيراً في التتمية الاقتصادية والاجتماعية، على الرغم من محدودية اهتمام الدولة بها،

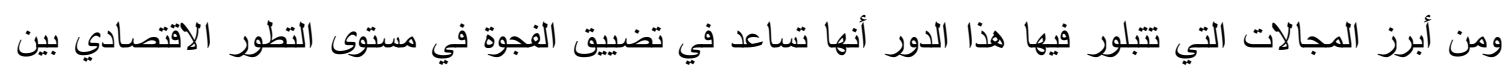

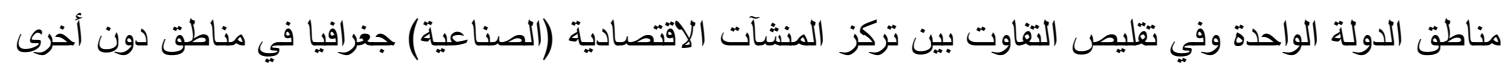
مما يحقق الترابط والتكامل بينهما، ويعمل التشابك الإنتاجي بين مختلف القطاعات، بل تتشر ثمار التتمية الاقتصادية في ربوع البلاد ونشر الرفاهية الاقتصادية وتحسين مستوى المعيشة (فرقش والزغيمي، 2011).

يشير (عبدالحميد، 2016) إلى أن المشاريع الصغرى تساعد على تخفيف الهجرة من الريف إلى المدينة لأنها تستخدم الموارد المحلية والقوى العاملة، مما يحقق التوازن الاجتماعي والاقتصادي الأفضل، وتساهم باستيعاب القوى العاملة وتقليل مستوى البطالة واكتساب المهارات من خلال ما يأتي:

1- تكلفة توفير فرصة العمل في المشاريع الصغرى هو أقل من نظيرتها في المشروعات المتوسطة والكبيرة، مما

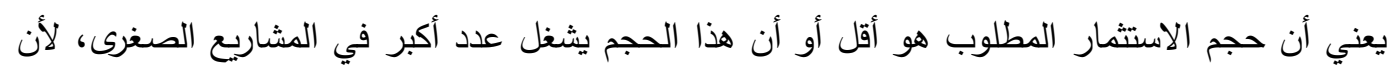
التكنولوجيا المستخدمة هي كثيفة العمالة. 2- للمشاريع الصغرى دور في إكساب المهارات للعاملين غير الماهرين وجعلهم عمالا ماهرين بمرور الوقت. 3- استيعاب العمالة الفائضة في الريف والقرى والمساعدة في خلق تجمعات للصناعات الريفية فيها. 4- 4تثغيل أفراد العائلة والأقارب والمعارف بثكل أسهل بسبب محدودية الإجراءات اللازمة للتعيين خاصة، وأن العمل قد يكون مؤقتا ووسيلة للحصول على فرصة عمل أفضل. 5- تطويع التكنولوجيا المتوسطة وتبسيطها للعاملين.

كما تؤدي المشاريع الصغرى إلى خلق فرص منتجة للعديد من المهارات والكفاءات، وتوسيع قاعدة رجال الأعمال،

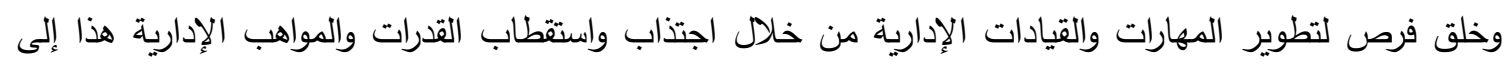

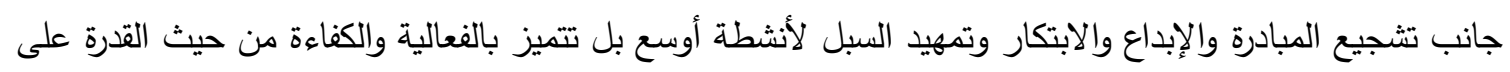
تحقيق الأهداف الاقتصادية لأصحابها أو من حيث قدرتها على إشباع رغبات واحتياجات العميل (برنوطي، 2016).

تعتبر المشاريع الصغرى وسيلة فعالة لتتويع وتوسيع القاعدة الإنتاجية وهيكل الإنتاج والتوزيع في الاقتصاد الوطني، إذ يمكن تكرار إنثائها في مناطق مختلفة من البلاد، كما تساعد على إحداث تتمية اقتصادية واجتماعية مما يؤثر

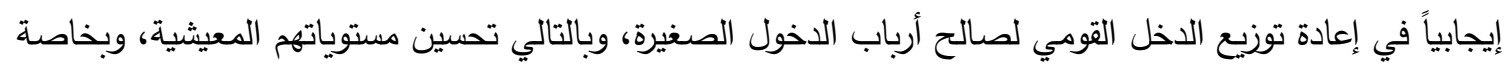

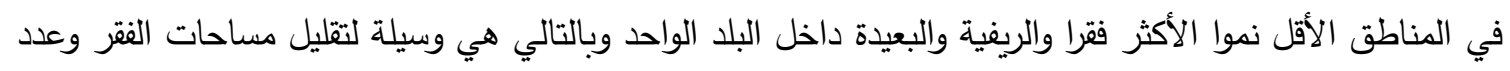
الأفراد الذي يقعون تحت حد الفقر (عثمان، 2014).

كذلك فان المشاريع الصغرى تساعد على تتمية الصادرات من خلال ما تقدمه من منتجات تامة صالحة للتصدير

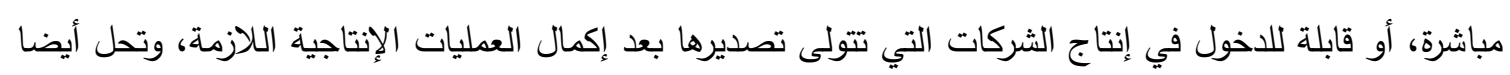
محل المنتجات المستوردة كإنتاج معوض وإحلال محل الواردات وبالتالي تقليل الإهدار في العملات الأجنبية، والنقد 
الأجنبي، إضافة إلى أنها تلبي الاحتياجات المحلية للسلع والخدمات محلياً بسبب تتويع تثكيلة ما تقدمه من مخرجات

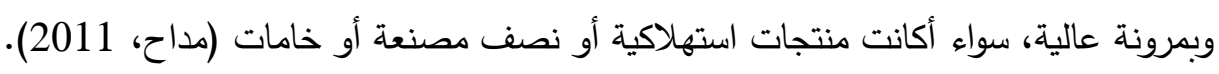

وتلعب المشاريع الصغرى دوراً في جذب المدخرات الصغيرة وتوسيع قاعدة التمويل الذاتي للمشاريع الصغرى، وعامل استقرار اقتصادي واجتماعي، وبخاصة أثناء الأزمات وفترات عدم الاستقرار والركود التي يواجهها الاقتصاد بين فترة وأخرى، فاتجاه الثباب للأعمال الجادة واستغلال طاقاتهم في أوجه تتموية بدلا من الأوجه الاستهلاكية التي تدفع الأنع

الشباب إلى انحرافات سلبية اقتصادية واجتماعية (عبد المطلب، 2015).

ويرى الباحث أن دور المشاريع الصغرى يؤدي إلى تحريك عجلة النمو الاقتصادي وفي تحقيق أهداف خطط التمية

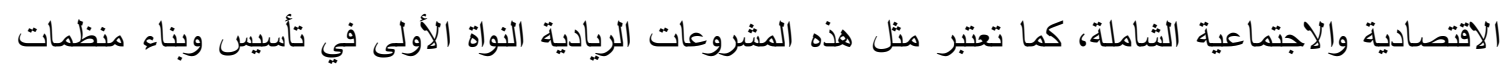

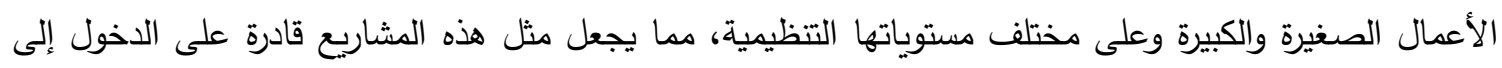
الأسواق، وهذه الحقائق تجعل المشاريع الخاصة من الحقول الهامة والواعدة في اقتصاديات الدول الصناعية المتقدمة منها والنامية على حد سواء.

منهجية البحث

اتبعت هذه الدراسة نوع الدراسات الميدانية المتضافرة بالأسلوب الوصفي التحليلي، مستهدفا تقييما صحيحا للمتطلبات

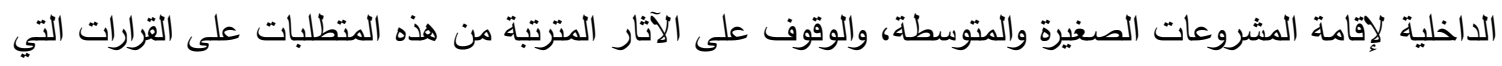
تتخذها المصارف الإسلامية لتقديم تمويلات لهذه المشروعات.

وقد تم اختيار وحدة التحليل والمعاينة من مجموع العاملين في المصارف الإسلامية الذين يشتغلون في دائرة الإيتمان،

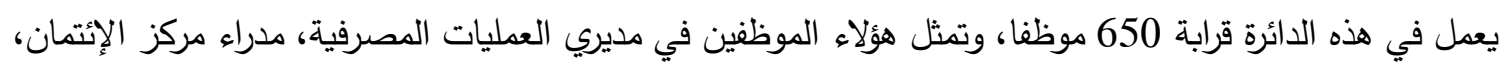

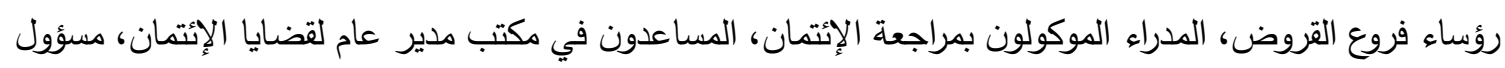
ضابط الإتتمان، المسؤول الرئيسي عن ضابط الإيتمان، المسؤول الرئيسي عن ضبط الإنسان، الإتمان، القائم على تحليل

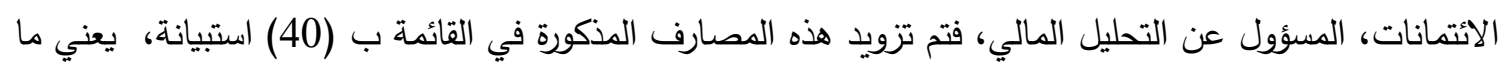

$$
\text { يصل إلى (240) استبيانة. }
$$

تم تصميم الاستبانة من قبل الباحث مراعيا المتغيرات التي يعتمد عليها، مع استعمال استفسارات تقييمية أكثر التصاقا

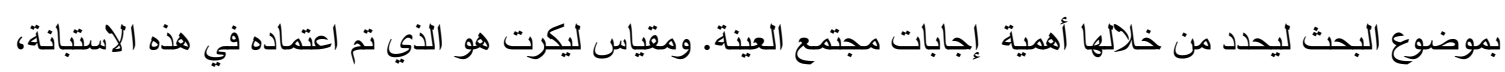
وهو المقياس الذي يتكون من خمس درجات يحدد من خلالها درجة الموافقة النسبية فيما يتعلق بفقرات الاستبانة (موافق بثدة 5 درجات، موافق 4 درجات، موافق إلى حد ما 3 درجات، غير موافق درجتين، غير موافق بشدة درجة

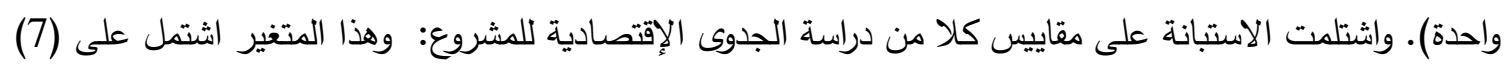

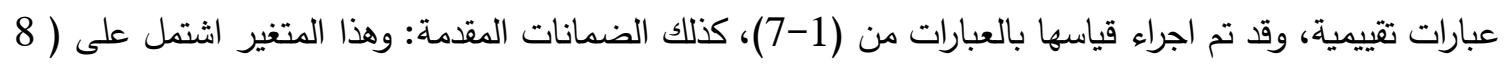

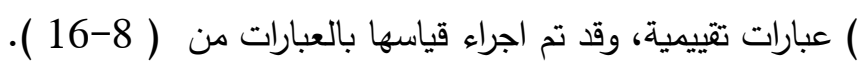

نتائج البحث

التحليل الوصفي للمشاركين هو الاختبار الأول الذي يتم تتفيذه للبحث الذي يجمع البيانات. يوفر هذا الاختبار صورة

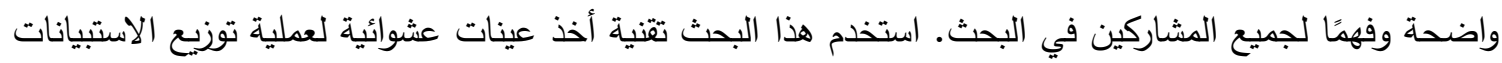


على عينة البحث. الهدف الرئيسي لاختبار التحليل الوصفي للمشاركين هو التأكد من أنه تم تتفيذ تقنية أخذ العينات

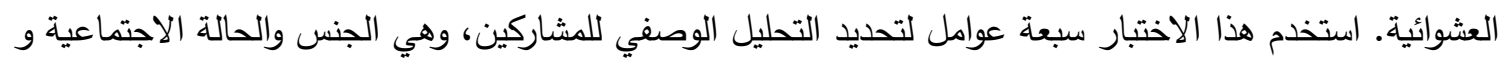
العمر والمستوى التعليمي و المنصب الوظيفي و سنوات الخبرة و المشاركة في التدريب. توضح النقاط والجداول

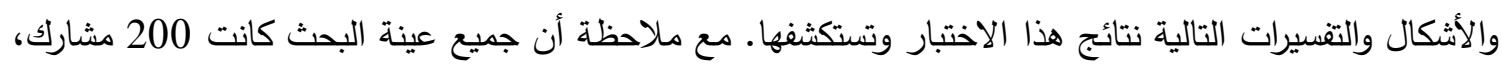
لذا فإن جميع النسب المئوية الواردة أدناه تعكس عدد العينات التي شاركت. الفئة الأولى من التحليل الوصفي للمشاركين هي فئة الجنس. كان لهذه الفئة فئتان فرعيتان كانتا مشاركتين في هذا البحث، هذه الفئات الفرعية من الذكور والإناث.

$$
\begin{aligned}
& \text { • بلغت نسبة الذكور 72.7٪ من إجمالي عدد عينة البحث وعددهم } 145 \text { مشارك. } \\
& \text { • شكلت النئة النسائية 27.3\% من إجمالي عدد عينة البحث وعددهم } 55 \text { مشاركة. }
\end{aligned}
$$

أظهرت النتائج أن غالبية المشاركين في البحث هم من الذكور الذين يمثلون 145 مشاركًا. يقدم الجدول 1 نتائج واضحة تم العثور عليها من هذه الفئة.

\begin{tabular}{|c|c|c|c|c|}
\hline \multicolumn{5}{|c|}{ الجنس } \\
\hline المجمعة النسبة المئوية & فعالة النسبة المئوية & النسبة المئوية & التكرار & المتغير \\
\hline 72.7 & 72.7 & 72.7 & 145 & |خكر \\
\hline \multirow[t]{2}{*}{100.0} & 27.3 & 27.3 & 55 & |نثى |ن | \\
\hline & 100.0 & 100.0 & 200 & |لاجمالي \\
\hline
\end{tabular}

جدول 2 :الجنس

الفئة الثانية من التحليل الوصفي للمشاركين هي الفئة العمرية. تضم هذه الفئة ست فئات فرعية شاركت في هذا

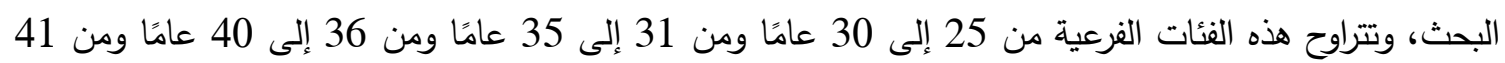

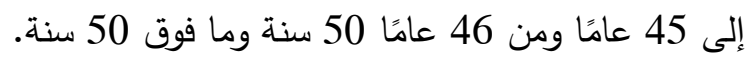

شكلت فئة المشاركين الذين تتراوح أعمارهم بين 25 و 30 سنة 9.9 \% و عددهم =20 من إجمالي عدد عينة البحث.

شكلت فئة المشاركين الذين تتراوح أعمارهم بين 31 و 35 عامًا 17.7٪ و عددهم =35 من إجمالي عدد عينة البحث. شكلت فئة المشاركين الذين تتراوح أعمارهم بين 36 و 40 سنة 31.2 ٪ و عددهم =62 من إجمالي عدد عينة البحث. شكلت فئة المشاركين الذين تتراوح أعمارهم بين 41-45 سنة 18.8 ٪ و عددهم =38 من إجمالي عدد

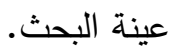
شكلت فئة المشاركين الذين تتراوح أعمارهم بين 46 و 50 عامًا 17.7٪ و عددهم =35 من إجمالي عدد

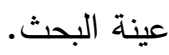


• شكلت فئة المشاركين الذين كانوا فوق سن 50 عامًا 4.6٪ و عددهم =9 من إجمالي عدد عينة البحث. يوضح الجدول التالي رقم 2 النتائج التي تم الحصول علئ عليها.

\begin{tabular}{|c|c|c|c|c|}
\hline المجمعة النسبة المئوية & فعالة النسبة المئوية & النسبة المئوية & التكرار & المتغير \\
\hline 9.9 & 9.9 & 9.9 & 20 & $25-30$ \\
\hline 27.7 & 17.7 & 17.7 & 35 & $31-35$ \\
\hline 58.9 & 31.2 & 31.2 & 62 & $36-40$ \\
\hline 77.7 & 18.8 & 18.8 & 38 & $41-45$ \\
\hline 95.4 & 17.7 & 17.7 & 35 & $46-50$ \\
\hline 100.0 & 4.6 & 4.6 & 9 & 50فما فوق \\
\hline & 100.0 & 100.0 & 200 & الاجمالي \\
\hline
\end{tabular}

كان لنوع المستوى التعليمي في هذا البحث ثلاث فئات هي الجامعي والماجستير والدكتوراه. كثفت النتائج المكتسبة ما يلي:

$$
\begin{aligned}
& \text { • كانت فئة الجامعي } 58.5 \text { \% مع عدد = } 117 \text { مشارك. } \\
& \text { • كانت فئة الماجستير } 23.0 \text { \% مع عدد = } 46 \text { مشارك. } \\
& \text { • وكان فئة الدكتوراه } 18.4 \text { \% مع عدد = } 37 \text { مشارك. } \\
& \text { • • الجدول رقم } 3 \text { يعرض النتائج. }
\end{aligned}
$$

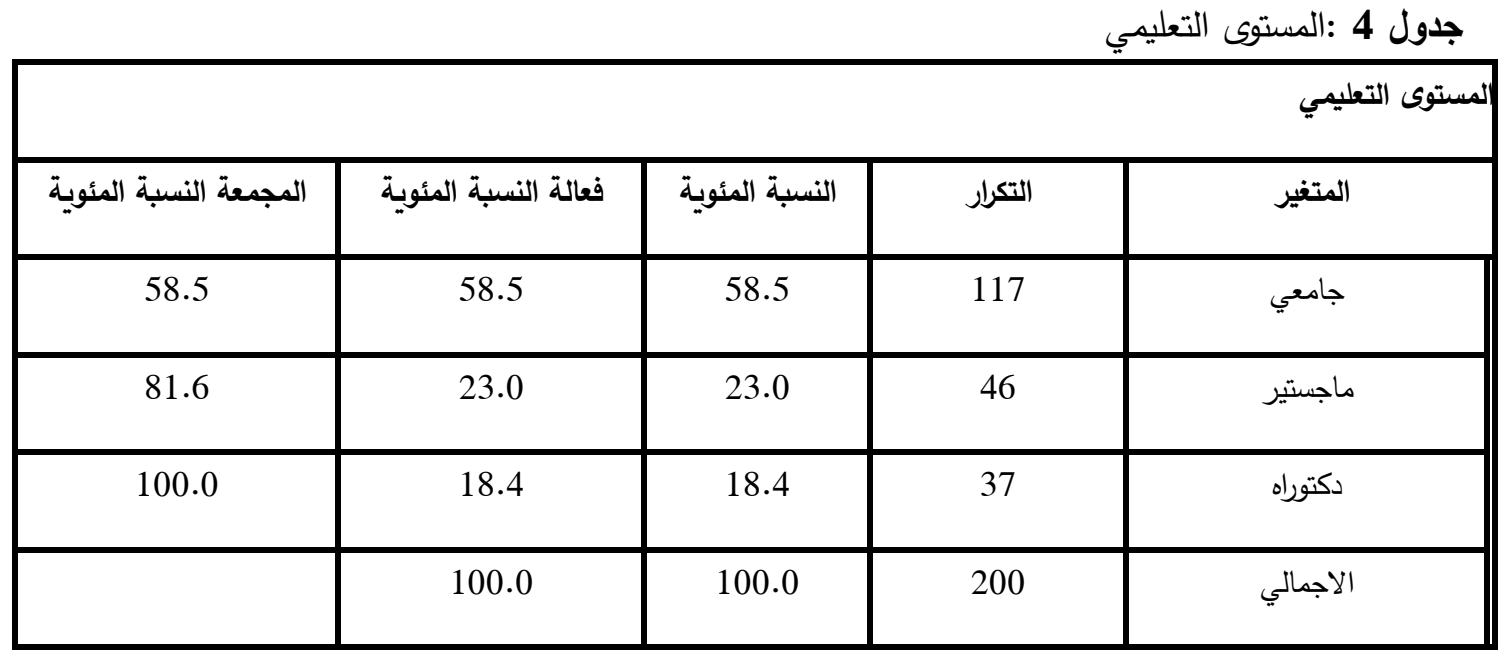

وقد تم تحليل أبعاد متطلبات إقامة المشروعات الصغيرة والمتوسطة تبعا لفقراتها: 
أولا: بعد دراسة الجدوى الإقتصادية للمشروع:

جدول 5 :المتوسطات الحسابية والانحرافات المعيارية لفقرات بعد دراسة الجدوى الإقتصادية للمشروع مرتبة تتازلياً

\begin{tabular}{|c|c|c|c|c|c|c|}
\hline الرتبة & المستوى & النسبة الأهمية & 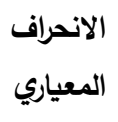 & المسابي & الفقرة & 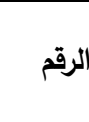 \\
\hline 3 & مرتفع & 75.60 & 0.56 & 3.78 & توفى التمويل من الدصرف الإسدوى الإسلامي يساعد في الحصول & FS.1 \\
\hline 1 & مرتفع & 77.40 & 0.51 & 3.87 & محتويات دراسة الجدوى الاقتصادية يساعد في التصوي الإسلامي & FS.3 \\
\hline 2 & مرتفع - & 76.00 & 0.55 & 3.80 & تراجع جهات مختصة في المصرف الإسلامي لدراسة & FS. 5 \\
\hline 4 & متوسط & 73.20 & 0.73 & 3.66 & خدمات التمويل المالي التي تعتمد على بيانات محددة تعلى الإمليم & FS.6 \\
\hline & مرتفع & 75.60 & 0.39 & 3.78 & الجدوى الإقتصادية للمشروع & \\
\hline
\end{tabular}

يظهر الجدول رقم 4 قيم المتوسطات الحسابية والانحرافات المعيارية لفقرات بعد دراسة الجدوى الإقتصادية للمشروع

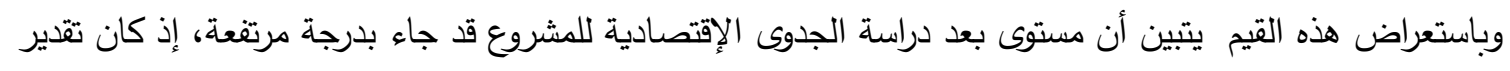

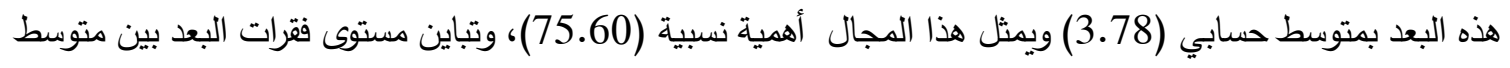
ومرتقع، حيث تراوحت قيم المتوسطات الحسابية بين (3.87 - 3.66)، وقد حقت الفقرة رقم (FS.3) الرتبة الأولى لئى وهي "محتويات دراسة الجدوى الاقتصادية يساعد في الحصول على التمويل من المصرف الإسلامي" اذ تم تقديرها بمتوسط حسابي (3.87) و يمثل هذا المتوسط أهمية نسبية (77.40)، كما حقتت الفقرة رقم (FS.6) المرتبة الأخيرة وهي "يعمل المصرف الإسلامي على تسريع أسلوب تقديم خدمات التمويل المالي التي تعتمد على بيانات محددة" حيث تم تقدير هذه الفقرة بمتوسط حسابي بقيمة (3.66) ويمثل هذا المتوسط أهمية نسبية بقيمة (73.20). 
ثانيا: بعد الضمانات المقدمة

جدول 6 :المتوسطات الحسابية والانحرافات المعيارية لفقرات بعد الضمانات المقدمة

\begin{tabular}{|c|c|c|c|c|c|c|}
\hline الرتبة & المستوى & الأهمية & المعياري & الحستوسط & الفقرة & الرقم \\
\hline 8 & متوسط & 68.60 & 0.63 & 3.43 & يطثدة مقابل الحصوف الإسلامي ضلى التمويل & WP.8 \\
\hline 3 & متوسط & 73.40 & 0.78 & 3.67 & يقيدمها المشروع اليب جديدة للمنتجات الإسلامي بابتكار طرق التذمات التي & WP. 10 \\
\hline 2 & مرتفع & 74.00 & 0.77 & 3.70 & الختلف الضمانات التي يطلبها المصرف & WP.11 \\
\hline 7 & متوسط & 70.60 & 0.66 & 3.53 & فيتخدم المصرف الإسلامي أساليب محددة & WP.12 \\
\hline 6 & متوسط & 71.40 & 0.81 & 3.57 & بحرص التمصريل المشروعات المتقدمة للحصلامي على المفاضلة على & WP.13 \\
\hline 3 & متوسط & 73.40 & 0.65 & 3.67 & 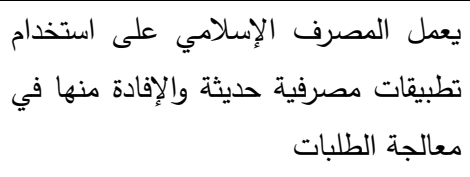 & WP.14 \\
\hline 1 & مرتفع & 74.80 & 0.82 & 3.74 & رعاية المصرف للمشروع تعتبر جزء من & WP. 15 \\
\hline 5 & متوسط & 72.00 & 0.77 & 3.60 & لبعض المشن يقدم المصرف الإسلامي تمويل & WP.16 \\
\hline & متوسط & 72.20 & 0.46 & 3.61 & الضمانات المقدمة & \\
\hline
\end{tabular}

يظهر الجدول رقم 5 قيم المتوسطات الحسابية والانحرافات المعيارية لفقرات بعد الضمانات المقدمة وباستعراض هذه القيم يتبين أن مستوى بعد الضمانات المقدمة قد جاء بدرجة متوسط، إذ كان تقدير هذه المجال بمتوسط حسابي (3.61) ويمثل هذا البعد أهمية نسبية (72.20)، وتباين مستوى فقرات البعد بين متوسط ومرتفع، حيث تراوحت قيم المتوسطات الحسابية بين (3.74 - 3.43)، وقد حقتت الفقرة رقم (WP.15) الرتبة الأولى وهي "رعاية المصرف للمشروع تعتبر جزء من الضمانات المقدمة لتمويله" اذ تم تقديرها بمتوسط حسابي (3.74) و يمثل هذا المتوسط أهمية 
نسبية (74.80)، كما حققت الفقرة رقم (Lotfaliei \& Lundberg) المرتبة الأخيرة وهي "يطلب المصرف الإسلامي ضمانات متثددة مقابل الحصول على التمويل" حيث تم تقدير هذه الفقرة بمتوسط حسابي بقيمة (3.43) ويمثل هذا المتوسط أهمية نسبية بقيمة (68.60)

كما هو موضح في الجدول التالي 6 فان قيم الالتواء والتثرطح لمتغيرات الدراسة مثلت بين القيم -3 و +3 كما اقترح بواسطة هاير (Hair, Sarstedt, Ringle, \& Mena, 2012)، الذي بدوره يؤكد خاصية التوزيع الطبيعي على هل مستوى المتغيرات.

جدول 7 : التوزيع الطبيعي على مستوى المتغيرات

\begin{tabular}{|c|c|c|}
\hline التفرطح & الالتواء & المتفيرات \\
\hline-.121 & -.301 & دراسة الجدوى الإقتصادية للمشروع \\
\hline .328 & -.518 & الضمانات المقدمة \\
\hline 1.204 & 1.052 & قرارات تمويل المشاريع الصغيرة والمتوسطة \\
\hline
\end{tabular}

يتبين من خلال الجدول 7 ان المتغيرات دراسة الجدوى الإقتصادية للمشروع والضمانات المقدمة على المشروع حصلت على نتائج مقبولة و منها قيم التباين المسموح 0.948، 0.865، 0.929، 0.881 على التوالي، الذي يشير الى لى

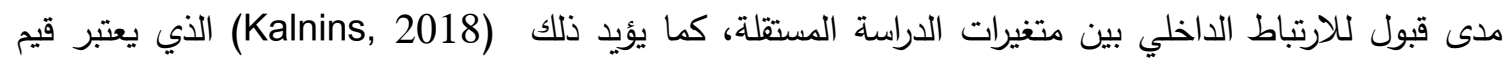
الارتباط الداخلي الاقل من 4.0 تعتبر مناسبة للتقدم في اختبار الفرضيات.

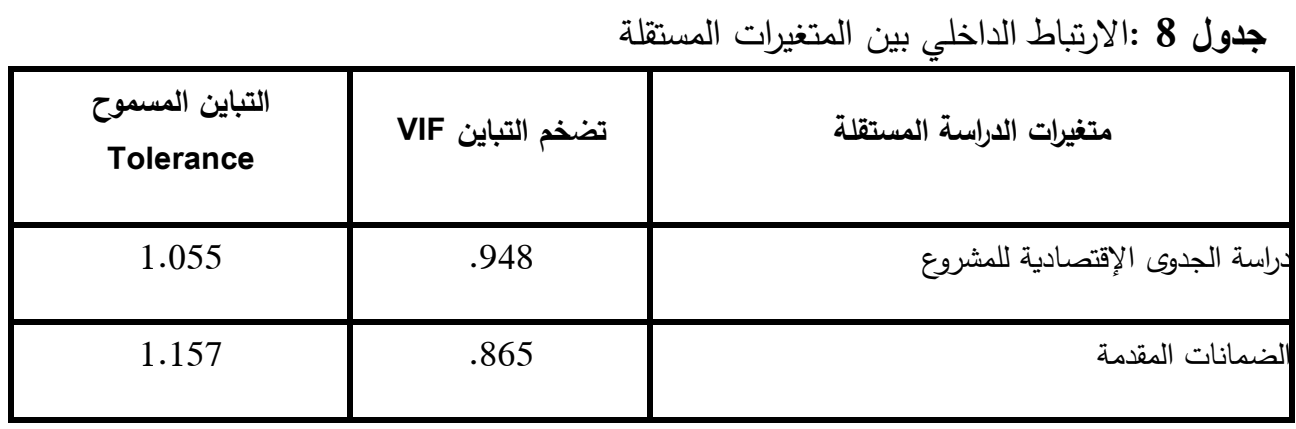

يهدف اختبار الارتباط لقياس الاتجاه بين متغيريين، من خلال اختبار العلاقة وتحديد اتجاها وهل تعتبر هذه العلاقة

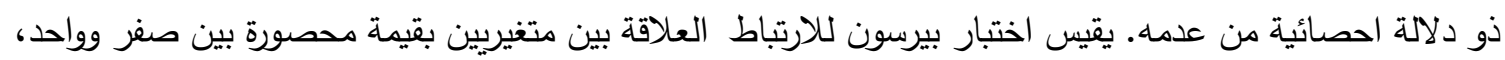
كلما اقتربت قيمة الارتباط للمتغيرييين الى الواحد كلما كانت الارتباط عالية جدا. يظهر الجدول 5.20 نتيجة اختبار

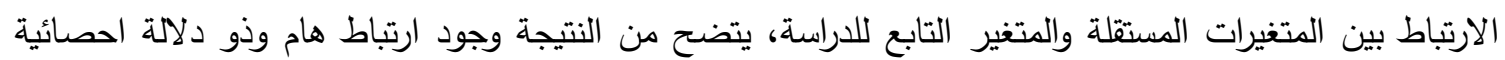
ايجابية بين المتغيرات المستقلة (دراسة الجدوى الإقتصادية للمشروع، الضمانات المقدمة) والمتغير التابع قرارات تمويل

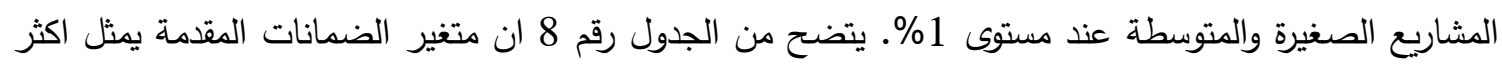

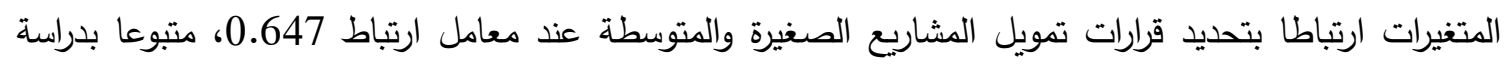

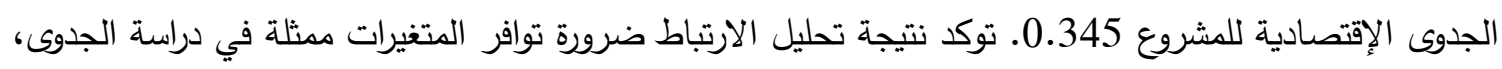


الضمانات المقدمة. ذلك ان دراسة الجدوى تكتسب اهميتها من خلال اظهار مدى ربحية المشروع من عدمه، الذي يقلل مخاطر الائتمان بالنسبة للبنك عند الموافقة عل التمويل. كلما زاد حجم الضمانات المقدمة من قبل طالب التمويل، كلما ساهم ذلك في التاثير على قرار قبول التمويل من البنك.

- correlation result جدول 9 :اختبار الارتباط

\begin{tabular}{|c|c|c|c|c|}
\hline 3 & 2 & 1 & & \\
\hline & & 1 & لمعامل ارتباط بيرسون & \multirow[t]{2}{*}{-راسة الجدوى الإقتصادية للمشروع } \\
\hline & & & Sig. (2-tailed) & \\
\hline & 1 & $.280^{* * *}$ & لمعامل ارتباط بيرسون & \multirow[t]{2}{*}{ ل لضمانات المقدمة } \\
\hline & & .000 & Sig. (2-tailed) & \\
\hline 1 & $.647^{* * *}$ & $.345^{* *}$ & لمعامل ارتباط بيرسون & \multirow[t]{2}{*}{ ترارات تمويل المشاريع الصغيرة والمتوسطة } \\
\hline & .000 & .000 & Sig. (2-tailed) & \\
\hline
\end{tabular}

(2-tailed). \%1 الارتباط هام وذو دلالة احصائية عند مستوى***.

الخلاصة

أظهرت النتائج وجود تأثير ذو دلالة إحصائية عند مستوى دلالة (0.05 م) لدراسة الجدوى الإقتصادية للمشروعات

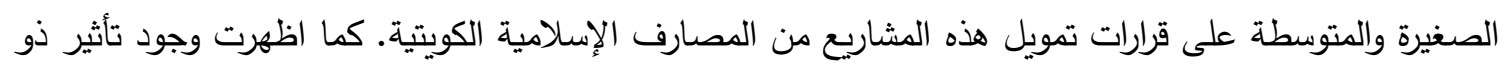
دلالة إحصائية عند مستوى دلالة (0.05 0.05$)$ للضمانات المقدمة من المشروعات الصغيرة والمتوسطة على قرارات

$$
\text { تمويل هذه المشاريع من المصارف الإسلامية الكويتية. }
$$

توكد النتائج المستقاه من التحليل الاحصائي اهمية الدور التي تلعبه دراسة الجدوى الاقتصادية في تثجيع المصارف

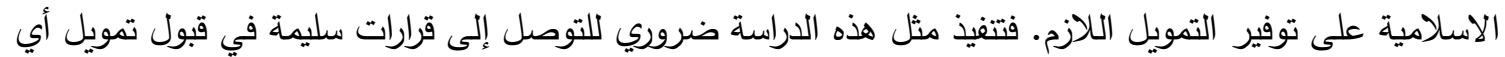

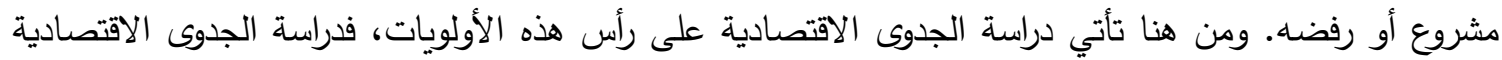

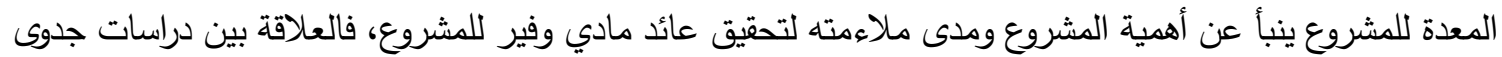
الاقتصادية والقرارت الاستثمارية والتمويلية يجب أن تكون علاقة دقيقة صحيحة لا تلعب فيها العواطف ولا الدراسات

$$
\text { الهشة غير مبنية على أسسس سليمة. }
$$

بالاظافة الى اهمية الضمانات وخصوصا للمصرف المانح للائتمان. حيث يعد معيار القدرة المالية للعميل أحد أهم المعايير التي تؤثر في مقدار المخاطر التي تتعرض لها إدارة الائتمان في البنك الإسلامي، حيث تتحدد هذه القدرة

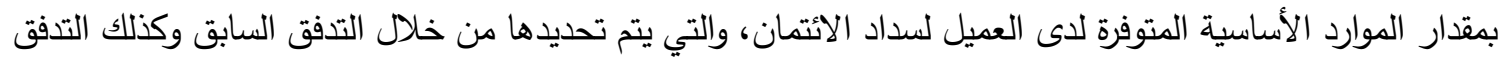
النقدي المتوقع في المستقبل، كما أنها تتمثل في حجم الديون الأخرى للعميل ومدى تمكنه من تحقيق الدخل الكافي لهني

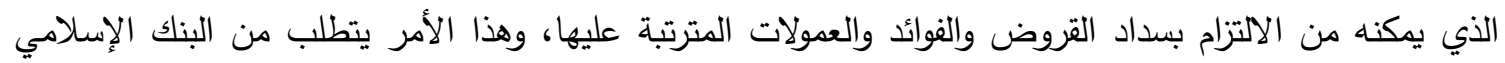

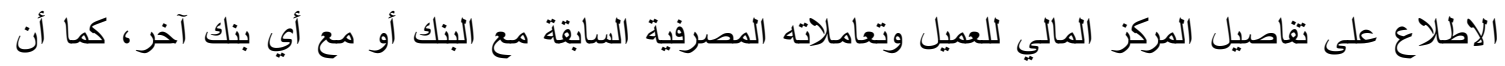

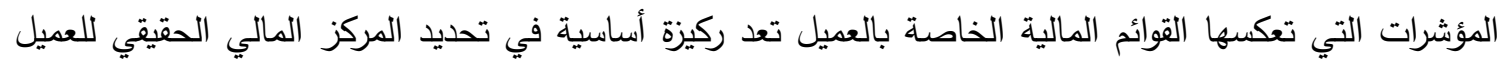
المقترض، فكلما كانت نتائج دراسة هذا الجانب إيجابية زاد اطمئنان متخذ القرار في البنك الإسلامي إلى قدرة المقترض محل الدراسة على سداد القرض المطلوب وفق الشروط المقترحة للقرض وفي مواعيد السداد التي سيتم الاتفاق عليها. 
توصي الدراسة قيام المصارف الإسلامية الكويتية بالمساهمة في إعتماد دراسات الجدوى الاقتصادية للمشروع الصغير

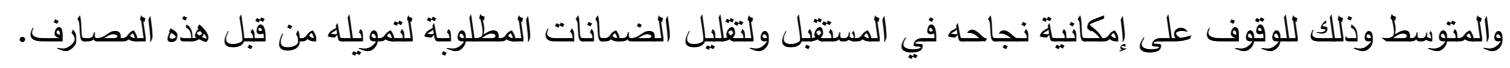

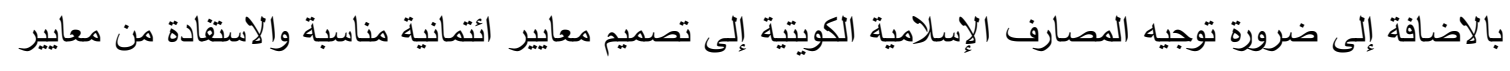

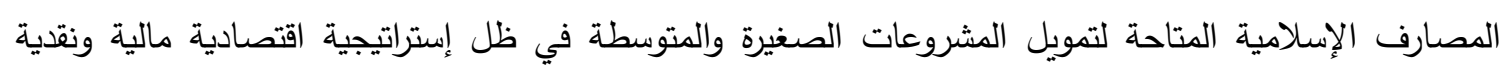

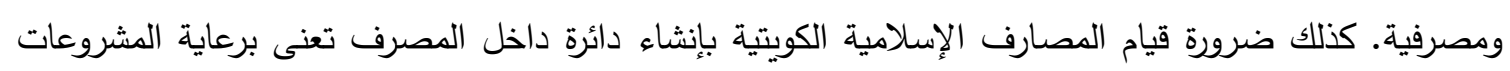
الصغيرة والمتوسطة بهدف توجيه أصحاب هذه المشاريع وتسهيل حصولها على التمويل.

المراجع أبو جليل، محمد منصور ، والعتوم، فراس، وهيكل، إيهاب، والكتبي، سعيد، (2017)، التسويق في المنشآت الصغيرة، دار غيداء للنشر والتوزيع، عمان، الأردن.

أبو دياب، نبيل، (2014). "تعريف المنشآت الصغيرة والمتوسطة وأهميتها في التنمية الاقتصادية والاجتماعية ومتطلبات نجاحها والمعوقات التي تواجهها". بحث مقدم إلى الملتقى السنوي الإسلامي السادس، الأكاديمية العربية للعلوم المصرفية، عمان، الأردن (27-29 - أيلول 2014).

برنوطي، سعاد نائف، (2016)، إدارة الأعمال الصغيرة (أبعاد للريادة)، دار وائل للنشر للطباعة والنشر والتوزيع، عمان، الأردن.

بودرامة، مصطفى، ومهملي، الوزناجي، (2010)، دور المشروعات المصغرة في تحقيق ريادة الأعمال في الجزائر، كلية العلوم الاقتصادية وعلوم التسيير، جامعة سطيف، الجمهورية، الجزائرية.

جواد، شوقي والمنصور ، كاسر ، (2017) إدارة المشروعات الصغيرة، دار الحامد للنشر والتوزيع، عمان، الأردن.

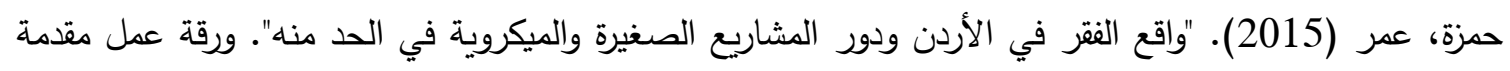

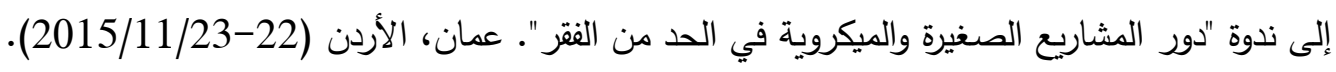
شرارة، ميساء حبيب، (2014)، الأثر التتموي للمشروعات الصغيرة الممولة في ظل إستراتيجية التتمية: (دراسة تطبيقية

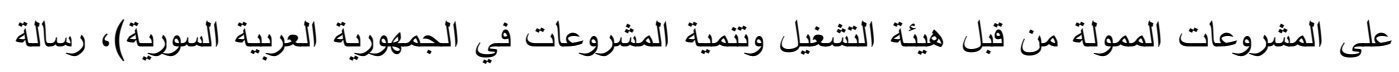
ماجستير، الأكاديمية العربية المفتوحة في الدنمارك، كلية الإدارة والاقتصاد.

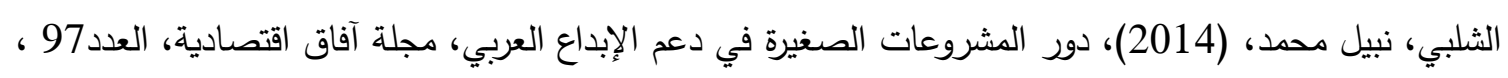

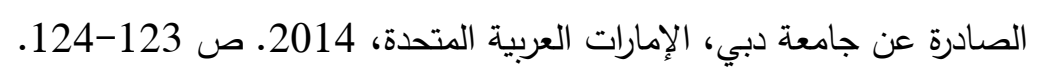
الصرايرة، رياض، (2016)، إنشاء وتطوير المؤسسات الصغيرة والمتوسطة في الأردن، ورقة عمل مقدمة إلى المؤتمر

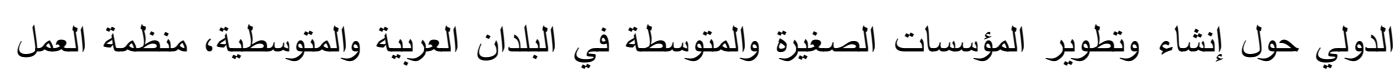

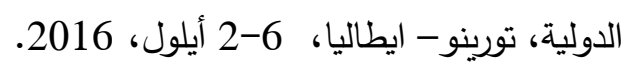

عبد الحميد، عبد المطلب، (2016)، إقتصاديات تمويل المشروعات الصغيرة، الدار الجامعية، الإسكندرية، مصر .

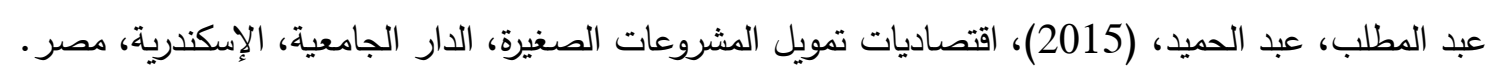

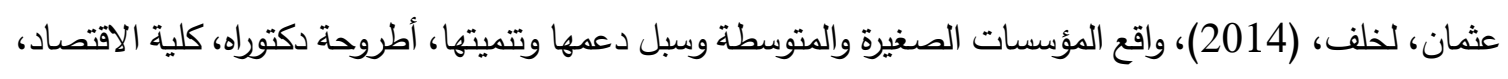
جامعة الجزائر ، الجزائر.

العيسوي، إبراهيم، (2016)، التتمية في عالم متغير ، دار الثروق للنشر والطباعة والتوزيع، عمان، الأردن.

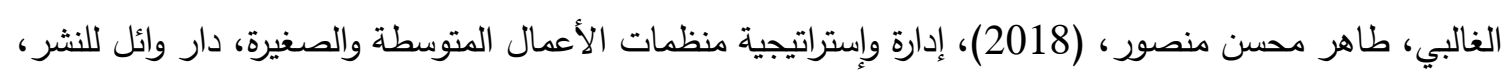
عمان، الأردن. 


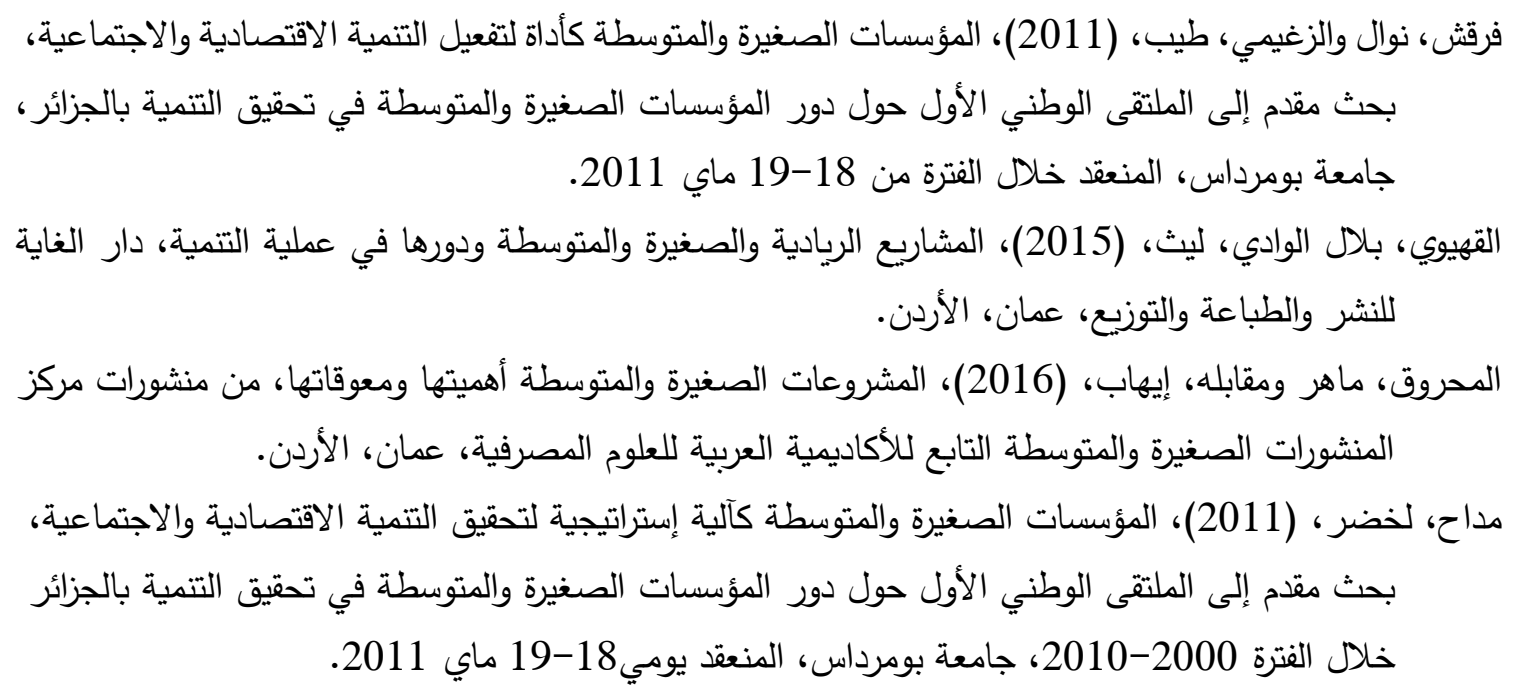

\section{References}

Abdel-Hamid, Abdel-Muttalib, (2016). Small Projects Finance Economics, University House, Alexandria, Egypt. (in Arabic)

Abdel-Muttalib, Abdel-Hamid, (2015). The Economics of Small Enterprise Finance, University House, Alexandria, Egypt. (in Arabic)

Abdullah, N. M. J. J. o. F. B. M. (2020). Family Entrepreneurship and Banking Support in Kuwait: Conventional Vs Islamic Banks. https://doi.org/10.1108/JFBM-06-2020$\underline{0049}$

Abu Diab, Nabil (2014). Defining Small and Medium Enterprises, their Importance in Economic and Social Development, the Requirements for their Success and the Obstacles they Face. Research presented to the Sixth Annual Islamic Forum, The Arab Academy for Banking Sciences, Amman, Jordan (27-29 - September 2014). (in Arabic)

Abu Jalil, Muhammad Mansour, Al-Atoum, Firas, Heikal, Ihab, and Al-Ketbi, Saeed, (2017). Marketing in Small Enterprises, Ghaida House for Publishing and Distribution, Amman, Jordan. (in Arabic)

Adams, B., \& Williams, K. R. J. A. E. J. M. (2019). Zone Pricing in Retail Oligopoly, 11(1), 124-156 .https://doi.org/10.1257/mic.20170130

Alareeni, B.A. (2019). The Associations between Audit Firm Attributes and Audit Quality-Specific Indicators: A Meta-Analysis, Managerial Auditing Journal, 34(1), 6-43. https://doi.org/10.1108/MAJ-05-2017-1559

Al-Ghalibi, Taher Mohsen Mansour, (2018). Management and Strategy of Medium and Small Business Organizations, Wael Publishing House, Amman, Jordan. (in Arabic)

Al-Issawi, Ibrahim, (2016). Development in a Changing World, Al-Shorouk Publishing, Printing and Distribution House, Amman, Jordan. (in Arabic)

Al-Mahrouq, Maher and his interview, Ihab, (2016). Small and Medium Enterprises their Importance and Constraints, published by the Small and Medium Publications Center of the Arab Academy for Banking Sciences, Amman, Jordan. (in Arabic)

Al-Qahwi, Bilal Al-Wadi, and Laith, (2015). Entrepreneurial, Small and Medium Enterprises and their Role in the Development Process, Dar Al-Ghaya for Publishing, Printing and Distribution, Amman, Jordan. (in Arabic)

Alqallaf, H. and Alareeni, B. (2018). Evolving of Selected Integrated Reporting Capitals among Listed Bahraini Banks, International Journal of Business Ethics and Governance, 1(1), 15-36. https://doi.org/10.51325/ijbeg.v1i1.10 
Al-Shalabi, Nabil Muhammad, (2014). The Role of Small Enterprises in Supporting Arab Creativity, Economic Horizons Magazine, published by the University of Dubai, United Arab Emirates, (97), 123-124. (in Arabic)

Barnouti, Suad Naif, (2016). Small Business Administration (Dimensions for Entrepreneurship), Wael Publishing House for Printing, Publishing and Distribution, Amman, Jordan. (in Arabic)

Bouderama, Mostafa, and Mahmeli, Woznaji, (2010). The role of microenterprises in achieving entrepreneurship in Algeria, Faculty of Economic Sciences and Management Sciences, University of Setif, Republic of Algeria. (in Arabic)

Farqash, Nawal and Zghaimi, Tayyib, (2011). Small and Medium Enterprises as a Tool to Activate Economic and Social Development, Research presented to the First National Forum on the Role of Small and Medium Enterprises in Achieving Development in Algeria, Boumerdes University, held during the period from 18-19 May 2011. (in Arabic)

Hair, J. F., Sarstedt, M., Ringle, C. M., \& Mena, J. A. (2012). An Assessment of the Use of Partial Least Squares Structural Equation Modeling in Marketing Research. Journal of the academy of marketing science, 40(3), 414-433. https://doi.org/10.1007/s11747-011-0261-6

Hamza, Omar (2015). The Reality of Poverty in Jordan and the Role of Small and MicroProjects in Reducing it. A working paper presented to the symposium on "The Role of Small and Micro Enterprises in Poverty Reduction". Amman, Jordan (22-23 / 11/2015). (in Arabic)

Jawad, Shawqi and Al-Mansour, Kasser, (2017). Small Projects Management, Al-Hamed House for Publishing and Distribution, Amman, Jordan. (in Arabic)

Kalnins, A. (2018). Multicollinearity: How common factors cause Type 1 errors in multivariate regression. Strategic Management Journal, 39(8), 2362-2385 https://doi.org/10.1002/smj.2783.

Lotfaliei, B., \& Lundberg, C. (2019). Reevaluating the Trade-off Theory of Capital Structure: Evidence from Zero-Leverage Firms. International Journal of Emerging Markets. https://doi.org/10.2139/ssrn.3478159

Maddah, Lakhdar, (2011). Small and Medium Enterprises as a Strategic Mechanism for Achieving Economic and Social Development, Research presented to the First National Forum on the Role of Small and Medium Enterprises in Achieving Development in Algeria during the period 2000-2010, Boumerdes University, held on 18-19 May 2011. (in Arabic)

Othman, Khalaf, (2014). The Reality of Small and Medium Enterprises and Ways to Support and Develop them, $\mathrm{PhD}$ Thesis, Faculty of Economics, University of Algiers, Algeria. (in Arabic)

Ramadhan, M., \& Girgis, M. (2018). Small and Medium Enterprises in Kuwait: Their

Impact and the Way Forward. Kuwait Institute of Scientific Research.

Sarayra, Riyadh, (2016). Establishment and Development of Small and Medium Enterprises in Jordan, Working Paper submitted to the International Conference on Establishing and Developing Small and Medium Enterprises in Arab and Mediterranean Countries, International Labor Organization, Turin - Italy, September 2-6, 2016. (in Arabic)

Sharara, Maysa Habib, (2014). The Development Impact of Small Projects Funded Under the Development Strategy: (An Applied Study on Projects Funded by the Operating and Enterprise Development Authority in the Syrian Arab Republic), Master Thesis, Arab Open Academy in Denmark, College of Management and Economics. (in Arabic) 